\title{
Chemoprotective effects of a recombinant protein from Pyropia yezoensis and synthetic peptide against acetaminophen-induced Chang liver cell death
}

\author{
YOUN HEE CHOI $^{1 *}$, EUN-YOUNG KIM $^{1 *}$, KOJI MIKAMI $^{2}$ and TAEK JEONG NAM ${ }^{1}$ \\ ${ }^{1}$ Institute of Fisheries Sciences, Pukyong National University, Busan 619-911, Republic of Korea; \\ ${ }^{2}$ Faculty of Fisheries Sciences, Hokkaido University, Hakodate 041-8611, Japan
}

Received April 2, 2015; Accepted June 16, 2015

DOI: $10.3892 /$ ijmm.2015.2253

\begin{abstract}
In the present study, the chemoprotective effects of recombinant Pyropia yezoensis (P. yezoensis) protein 1 (PYP1) were examined in acetaminophen (APAP)-treated Chang liver cells. The analysis of $P$. yezoensis revealed the presence of both mature and immature variants of PYP1. PYP1s, designated as PYP1 (15 kDa), PYP1-AC (12 kDa) and PYP1-B (5 kDa), were successfully expressed in Escherichia coli, and their chemoprotective effects were then examined. In addition, a peptide of 11 residues (ALEGGKSSGGG), which is a common sequence at the N-terminus all of the PYP1s, was synthesized and examined. The effects of treatment with PYP1s and the synthetic peptide (SP) on cell proliferation were determined by MTS assay. Our results clearly demonstrated that treatment with all the PYP1s and SP significantly promoted the proliferation of Chang liver cells, protecting them against APAP. Thus, we concluded that recombinant PYP1s exert protective effects against injury to Chang liver cells.
\end{abstract}

\section{Introduction}

Seaweed comprises abundant bioactive antioxidants, soluble dietary fibers, proteins, minerals, vitamins, phytochemicals and polyunsaturated fatty acids (1). It has been reported that these bioactive components (e.g., fucoxanthin, fucoidan and other extracts) exert a variety of effects in humans, such as anti-obesity (2), anticoagulant (3) and antitumor effects (4). The value of these properties as a function of bioactive substances in seaweed has been studied with regards to food,

Correspondence to: Professor Taek Jeong Nam, Institute of Fisheries Sciences, Pukyong National University, 474 Ilgwang-ro, Ilgwang-myeon, Gijang-gun, Busan 619-911, Republic of Korea E-mail: namtj@pknu.ac.kr

*Contributed equally

Key words: Pyropia yezoensis, recombinant protein, chemoprotective effect, acetaminophen-induced cell death pharmaceuticals and medicinal purposes (1). In particular, Hizikia fusiformis extract was found to protect against injury to the liver and stomach in rats; this was proven by studying its molecular mechanisms of action $(5,6)$. Moreover, it has been reported that a glycoprotein from Pyropia yezoensis (P. yezoensis) exerts anti-inflammatory effects in RAW 264.7 mouse macrophages (7). In addition, a protein isolated from $P$. yezoensis was shown to exert chemoprotective effects on acetaminophen (n-acetyl-p-aminophenol, APAP)-induced liver injury in rats (8). APAP is an over-the-counter drug that is widely used for its analgesic and antipyretic effects. APAP is safe when used at therapeutic levels; however, an acute or cumulative overdose can cause severe liver injury and possibly liver failure (9). Studies on APAP toxicity have been investigated both in vitro (10-13) and in vivo $(8,14-17)$. The ability of seaweed to exert chemoprotective effects against APAP means they are advantageous to various organisms.

Recently, the association between the molecular structure and function of seaweed was reported (18). Choi etal(18) reported that the synthetic peptide (SP) ALEGGKSSGGGEATRDPEPT, which is present at the $\mathrm{N}$-terminus of mature $P$. yezoensis protein 1 (PYP1), demonstrated chemoprotective effects against APAP-induced Chang liver cell death.

In the present study, 3 proteins (PYP1, PYP1-AC and PYP1-B) were derived from the cDNA that encodes PYP1 and were then investigated for their chemoprotective effects against APAP-induced Chang liver cell injury. Moreover, the N-terminal 11 residue sequence of SP, ALEGGKSSGGG, which represents a common sequence among all 3 peptides, was synthesized and compared with the PYP1s.

\section{Materials and methods}

Molecular cloning of cDNA and the gene encoding PYP1. To determine the N-terminal amino acid sequence of PYP1 (18), the $P$. yezoensis expressed sequence tag (EST) database of the Kazusa DNA institute (Chiba, Japan) was surveyed. Since the resultant information indicated that both mature and immature mRNAs were present, cDNA encoding these variants was cloned. Briefly, the cultivation of $P$. yezoensis gametophytes and the amplification of cDNA from total RNA were performed as previously described by Uji et al (19). DNA fragments corre- 
sponding to the open reading frames (ORFs) of mature and immature variants were then amplified by polymerase chain reaction (PCR) using the following primer sets: PYP1-F and PYP1-R, PYP1-F and PYP1-AC-R, and PYP1-F and PYP1-B-R (Table I). The PCR conditions were as follows: 30 cycles at $98^{\circ} \mathrm{C}$ for $10 \mathrm{sec}$ and $68^{\circ} \mathrm{C}$ for 2 min using PrimeSTAR HS DNA polymerase with GC buffer (Takara Bio., Otsu, Japan). Separation, purification, cloning and sequence analysis were performed as previously described by Uji et al (19), with the exception of the $\mathrm{pENTR/SD/D-TOPO} \mathrm{vector} \mathrm{(Invitrogen/Life} \mathrm{Technologies,}$ Carlsbad, CA, USA), which was used for the cloning and construction of plasmids that were expressed in bacteria as entry plasmids. To isolate the genomic fragment containing PYP ORF information, genomic DNA was prepared from gametophytes using a DNeasy Plant Mini kit (Qiagen, Hilden, Germany), and genomic PCR was performed as described above, using the PYP1-F and PYP1-R primers. The amplified fragment was inserted into a pCR-Blunt II-TOPO cloning kit (Clontech Laboratories, Inc., Mountain View, CA, USA) and sequenced.

Expression analysis. To analyze the expression profile of the PYPl gene in both gametophytes and sporophytes of $P$. yezoensis, several generations were cultured and used for total RNA extraction in order to amplify the cDNA, as previously described by Uji et al (19). Following the synthesis of the first-strand cDNA using a PrimeScript II First Strand cDNA Synthesis kit (Takara Bio), reverse-transcription PCR (RT-PCR) was performed using Phusion High-Fidelity DNA polymerase (New England BioLabs, Inc., Beverley, MA, USA) with the primer sets described above, under the following conditions: $98^{\circ} \mathrm{C}$ for $1 \mathrm{~min}$ and 30 cycles at $98^{\circ} \mathrm{C}$ for $10 \mathrm{sec}$, $55^{\circ} \mathrm{C}$ for $30 \mathrm{sec}$, and $72^{\circ} \mathrm{C}$ for $1 \mathrm{~min}$.

Construction of PYPI expression plasmids. Gateway Technology (Invitrogen/Life Technologies) was employed to construct the expression plasmids for the PYP1 and PYP1 variants in Escherichia coli (E. coli). To produce the destination vector, pQE-82L (Qiagen) was digested with SmaI in the multicloning site and ligated using Restriction fragment analysis (RFA; Invitrogen/Life Technologies). The resultant plasmid was designated as pQE80L-DES. LR recombination reactions were then performed with entry plasmids and pQE82L-DES according to the manufacturer's instructions, thereby, producing pQE82L-PYP1, pQE82L-PYP1-AC and pQE82L-PYP1-B.

Overexpression and purification of recombinant $P Y P 1$, PYP1-AC and PYP1-B. Plasmids (pQE82L-PYP1, pQE82LPYP1-AC and pQE82L-PYP1-B) were transformed into the $E$. coli strain DH5 $\alpha$ and incubated on ice for $30 \mathrm{~min}$. S.O.C medium (200 $\mu \mathrm{l}$; Invitrogen Life Technologies) was added followed by incubation for $1 \mathrm{~h}$ at $37^{\circ} \mathrm{C}$. The mixture was then spread on a plate with LB medium containing $100 \mu \mathrm{g} / \mathrm{ml}$ ampicillin and incubated for $16 \mathrm{~h}$ at $37^{\circ} \mathrm{C}$. After confirming the transformation in $E$. coli DH5 $\alpha$ cells, the plasmids were transformed into the E. coli strain BL21 (DE3) in a similar manner.

For the induction of the expression of PYP1, PYP1-AC and PYP1-B, LB medium $(20 \mathrm{ml})$ containing $100 \mu \mathrm{g} / \mathrm{ml}$ ampicillin was inoculated and grown at $37^{\circ} \mathrm{C}$ overnight. Cultures were inoculated into $1 \mathrm{ml}$ of LB medium containing ampicillin $(100 \mu \mathrm{g} / \mathrm{ml} ; 1: 50)$ with fresh LB culture. The cultures were
Table I. Primers used for PCR.

\begin{tabular}{ll}
\hline Primer & \multicolumn{1}{c}{ Sequence } \\
\hline PYP1-F & 5'-CACCATGGCGTTCGTGTCTGGGTTCAC-3' \\
PYP1-R & 5'-CTTGCCCTCAGCCTTCTTCTTG-3' \\
PYP1-AC-R & 5'-GTACGAGCGCGAGGTTGCGG-3' \\
PYP1-B-R & 5'-ACGTACCGGCTCAGGGTCAC-3' \\
\hline
\end{tabular}

PYP1 Pyropia yezoensis protein 1.

grown at $37^{\circ} \mathrm{C}$ until an optical density (OD) ${ }_{600}$ of 0.5 was reached. One milliliter of sample was taken immediately prior to induction, and expression was induced by the addition of isopropyl $\beta$-D-1-thiogalactopyranoside (IPTG) to a final concentration of $1 \mathrm{mM}$. The cells were harvested by centrifugation at 4,000 x g for $20 \mathrm{~min}$, resuspended in binding buffer (20 mM Tris-HCl, pH 8.0) and lysed by ultrasonic disruption (Sonics \& Materials Inc., Newtown, CT, USA). The sonicated extracts were then separated into soluble and insoluble fractions by centrifugation at $4,000 \mathrm{x}$ g for $20 \mathrm{~min}$ at $4^{\circ} \mathrm{C}$.

To purify the recombinant proteins, soluble fractions containing the PYP1s were loaded onto an Ni-NTA column (Qiagen) with Ni-NTA His•Bind Resin (Merck Millipore, Darmstadt, Germany) using lysis buffer $\left(50 \mathrm{mM} \mathrm{NaH}{ }_{2} \mathrm{PO}_{4} \cdot \mathrm{H}_{2} \mathrm{O}\right.$, $300 \mathrm{mM} \mathrm{NaCl}$ and $10 \mathrm{mM}$ imidazole). After complete loading, weakly bound proteins were removed with wash buffer $(50 \mathrm{mM}$ $\mathrm{NaH}_{2} \mathrm{PO}_{4}, 300 \mathrm{mM} \mathrm{NaCl}$ and $20 \mathrm{mM}$ imidazole). Proteins were subsequently eluted from a Ni-NTA spin kit (Qiagen) with elution buffer $\left(50 \mathrm{mM} \mathrm{NaH} \mathrm{PO}_{4}, 300 \mathrm{mM} \mathrm{NaCl}\right.$ and $250 \mathrm{mM}$ imidazole), according to the manufacturer's instructions. The concentrations of the protein samples collected during purification and purified samples were measured using the BCA method using a BCA protein assay kit (Pierce Biotechnology, Rockford, IL, USA).

All protein samples were resolved by $17 \%$ sodium dodecyl sulfate polyacrylamide gel electrophoresis (SDS-PAGE). For $\mathrm{N}$-terminal sequencing analysis, proteins were transferred onto polyvinylidene fluoride (PVDF) membranes (Millipore, Billerica, MA, USA) using an electrophoresis power supply (GE Healthcare Bio-Sciences AB, Uppsala, Sweden).

Peptide synthesis. The N-terminal 11-residue sequence, which was a common sequence in all the PYP1s (ALEGGKSSGGG), was synthesized by Peptron (Daejeon, Korea). Purification of the SP was performed on a Shimadzu Prominence HPLC system and controlled using the software package Class-VP, 6.14 with a C18 column (Shiseido Capcell Pak; Shiseido, Tokyo, Japan) in $0.1 \%$ trifluoroacetic acid (TFA)/water and a gradient of $10-70 \%$ acetonitrile in $0.1 \%$ TFA, at a flow rate of $1 \mathrm{ml} / \mathrm{min}$ and ultraviolet (UV) detection at $220 \mathrm{~nm}$. The molecular mass was confirmed at $918 \mathrm{Da}$ (it matched the sequence mass) using mass analysis (HP 1100 Series LC/MSD; Agilent Technologies, Inc., Santa Clara, CA, USA).

Cell culture. The Chang liver cell line (HPV-18) was obtained from the American Type Culture Collection (ATCC, Rockville, MD, USA). The cells were cultured in minimum essential 

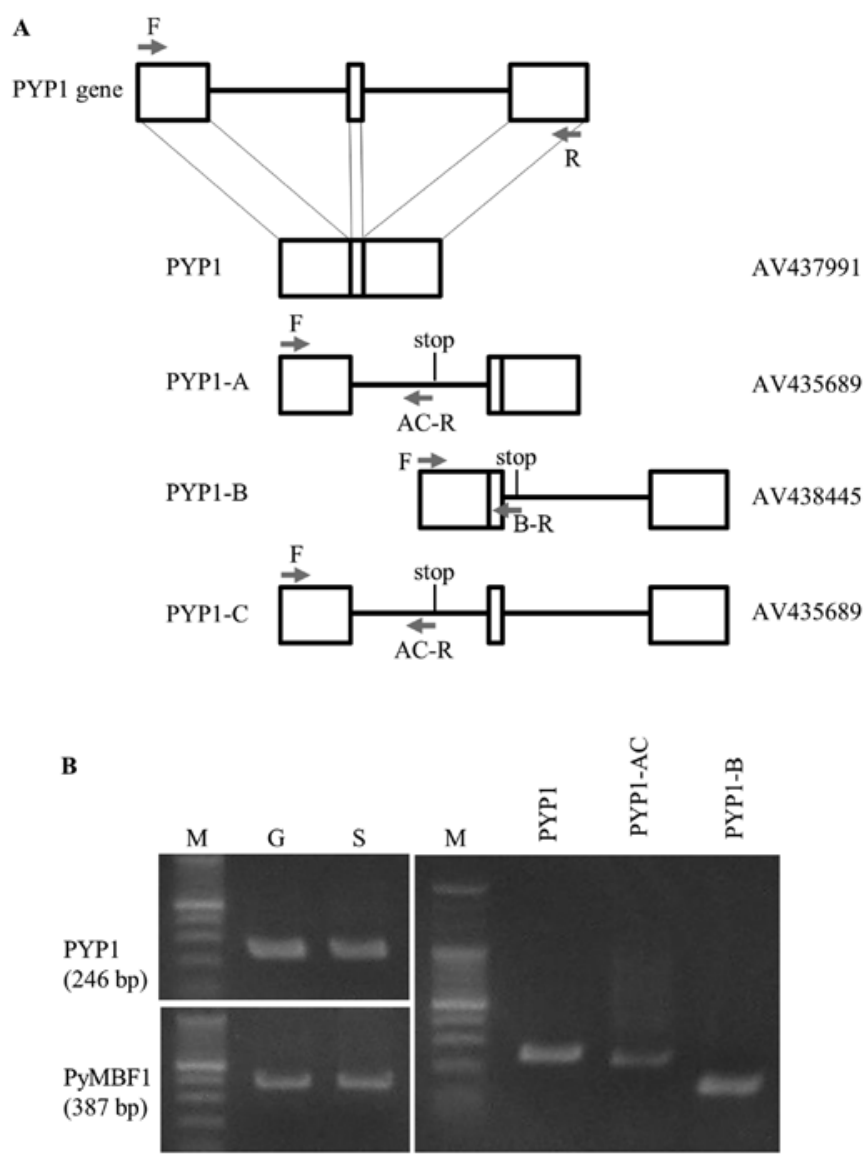

Figure 1. Identification and expression of mature and immature Pyropia yezoensis protein 1 (PYPl) gene transcripts. (A) Schematic representation of PYP1 and its variants produced by complete and alternative splicing, respectively, compared with the genomic organization of the PYP1 gene. Empty boxes represent exons, while bars represent introns. Positions of stop codons are indicated in the schemes of each variant. AV numbers determined by the Kazusa DNA Research Institute are names of clones whose sequences were determined previously. Arrows indicate the positions of primers used for RT-PCR; (Table I): F, PYP1-F; R, PYP1-R; AC-R, PYP1-AC-R; B-R, PYP1-B-R. (B) Gel images showing the results from RT-PCR. Comparison of $P Y P 1$ gene expression between gametophytic $(\mathrm{G})$ and sporophytic (S) generations (left panel). The expression pattern of $P y M B F 1$ (19) is presented as a reference. The presence of transcripts from the mature form and splice variants in the gametophytic generation can be noted (right panel). The sizes of the RT-PCR fragments for PYP1, PYP1-AC and PYP1-B are 246, 232 and $141 \mathrm{bp}$, respectively. $\mathrm{M}$, protein marker.

medium (MEM) supplemented with 10\% fetal bovine serum (FBS; HyClone, Logan, UT, USA), $100 \mathrm{U} / \mathrm{ml}$ penicillin and $100 \mathrm{mg} / \mathrm{ml}$ streptomycin. The cultures were maintained in a humidified incubator at $37^{\circ} \mathrm{C}$ in $5 \% \mathrm{CO}_{2}$. The medium was replaced every 2 days.

Cell proliferation assay. The effects of PYP1, PYP1-AC, PYP1-B and SP treatment on cell proliferation in the cells treated with $15 \mathrm{mM}$ APAP were colorimetrically determined by MTS assays using CellTiter 96 AQueous One Solution reagent (Promega, Madison, WI, USA). The cells were seeded in 96-well plates at a density of $1.5 \times 10^{5}$ cells/well. Following incubation for $24 \mathrm{~h}$, the attached cells were maintained in serum-free medium (SFM) for $6 \mathrm{~h}$, and this was followed by treatment with PYP1, PYP1-AC, PYP1-B or SP $(0-1,000 \mathrm{pg} / \mathrm{ml})$ for an additional $24 \mathrm{~h}$. The cells were then incubated with MTS solution at $37^{\circ} \mathrm{C}$ for $30 \mathrm{~min}$, and the absorbance was measured at $490 \mathrm{~nm}$ using a microplate reader (BioTek Instruments, Inc., Winooski, VT, USA). The $\mathrm{OD}_{490}$ values of the control cells were designated as $100 \%$.

4,6-Diamidio-2-phenylindole (DAPI) staining assay. The cells were washed twice with phosphate-buffered saline (PBS) and fixed with $4 \%$ paraformaldehyde. The fixed cells were incubated at $37^{\circ} \mathrm{C}$ for $20 \mathrm{~min}$ and washed twice with PBS. The cells were then stained with $1 \mu \mathrm{g} / \mathrm{ml}$ DAPI and incubated at room temperature for $20 \mathrm{~min}$ in the dark. The stained cells were observed under a fluorescence microscope (ECLIPSE TS100-F; Nikon Corp., Tokyo, Japan).

Statistical analysis. Data were evaluated by one-way analysis of variance (ANOVA) using the Statistical Package for the Social Sciences version 10.0 (SPSS, Inc., Chicago, IL, USA). Significant differences between means were identified using Duncan's multiple range test $(\mathrm{P}<0.05)$. A $\mathrm{P}$-value $<0.05$ was considered to indicate a statistically significant difference.

\section{Results}

Identification and characterization of multiple PYP1 mRNA transcripts. The SP, ALG EGKSSGGGEATRDPEPT, corresponding to the N-terminus of mature PYP1, has been shown to exhibit chemoprotective activity against APAP-induced Chang liver cell death (18). In order to obtain a full-length cDNA encoding PYP1 for use in inducing expression in bacteria, the P. yezoensis EST database of the Kazusa DNA Research Institute (http://est.kazusa.or.jp/en/plant/porphyra/EST/) was surveyed. A comparison of nucleotide sequences from the genomic PYPI gene revealed the presence of non-spliced introns, which were determined to cause the various lengths of PYP1 mRNAs (Fig. 1A), indicating that alterative splicing produces mature and immature $P Y P 1$ mRNAs.

As shown in Fig. 1A, the PYPl gene contains 2 introns; thus, the coding region is divided into 3 exons. Three types of immature cDNA contain either the first or second intron, or both introns. Proteins derived from the cDNA containing the first, second, or both introns were designated as PYP1-A, PYP1-B and PYP1-C, respectively (Fig. 1A). PYP1-A and PYP1-C encode the same protein, as they both contain the first intron, resulting in 3 PYP1 proteins: one is mature and the other two are variants, but all share an amino acid sequence corresponding to the first exon (Fig. 2). We refer to this mixture of PYP1-A and PYP1-C as PYP1-AC.

RT-PCR was employed to examine the expression of the $P Y P 1$ gene in gametophytic and sporophytic generations of $P$. yezoensis. Since a homology search of $P$. yezoensis in the EST database resulted in ESTs derived from mRNAs of the gametophytic generation being found (data not shown), the generation-specific expression of the $P Y P 1$ gene was speculated. However, Fig. 1B (left panels) clearly illustrates the expression of the PYPl gene in the gametophytic and sporophytic generations. Moreover, the presence of transcripts corresponding to PYP1-AC and PYP1-B was confirmed by RT-PCR (Fig. 1B, right panel), thus indicating that $P$. yezoensis-treated cells may contain all 3 PYP1 proteins. 
1 ATGGCGTTCGTGTCTGGGTTCACGGGCATGCCCGTGACGGCCCGCGTGTCCAAGGCGGTG

61 TGCCGCACGCGCATGGGGCTGGAGGGCGGCAAGTCCTCGGGGGGGGGTATGTTGTTGTCC

121 CAGCTTTACTGCGGCCCACTCGGGTCGGTGCCCGCTTACCAGCGATGTTGTCGTTCTATA

181 GATGTATCCGCATCTGGTGGTGGAAGCGCCGCAACCTCGCGCTCGTACTGACCCCTGCTG

241 CCACTCTTACGTTGCTGTGCACGTGTGTCGTTTTGCTTCACTCTGGCTGCCGATTGTATG 301 TGCAGGTGAGGCCACCCGTGACCCTGAGCCGGIACGITGAGTCTCAAGCTCAGGATGTAT 361 TCGTGGTCTGCCGACCAAATCTGCGCGAGGGGGTACTGACGGGGTGTCAGGGTTGTGGAC 421 GCTGAATGTGCCAGACGAGATGTATTCAAGCGCTGACGGATCCTCCCTGTCTTCCACTGT 481 GTGCCTGTGTGCATGCATCTGCCTTCTACGCTATGTCCACAGACCGCTGTTGACCCCAAC 541 GATCCGAAGGGGAGCAGCAGGCCATCCACGTGGCCCCGTCGTTCGCCGACTACCTGAAG 601 GCGCAGGCGGACAAGAAGAAGGCTGAGGGCAAGTAA 636

Figure 2. Exon-intron structure of Pyropia yezoensis protein 1 (PYPl) gene. The nucleotide sequence of $P Y P 1$ is presented, and exons are highlighted. Exon-intron junctions fit the GT-AG rule.

Preparation of bacterially expressed PYPl proteins. To evaluate the functional activities of the 3 PYP1 proteins, PYP1, PYP1-AC and PYP1-B plasmids for the induction of expression in bacteria were constructed, which produced proteins with a $6 \mathrm{xHis}$ tag.

Expression and purification of PYP1,PYP1-AC and PYP1-B.The results of SDS-PAGE revealed purified soluble proteins (Fig. 3). PYP1, PYP1-AC and PYP1-B were first purified with Ni-NTA His Bind Resin (Merck Millipore) (Fig. 3A-a, B-a and C-a). PYP1, PYP1-AC and PYP1-B were then further purified using a Ni-NTA Spin kit (Qiagen), which produced proteins with the following molecular weights: 15,12 and $5 \mathrm{kDa}$, respectively (Fig. 3A-b, B-b and C-b). This two-step process used to extract PYP1s was effective.

Physiological activity of PYP1, PYP1-AC and PYP1-B. The effects and toxicity of PYP1, PYP1-AC and PYP1-B on Chang liver cells were determined by MTS assays. As shown in Fig. 4A, PYP1 was non-toxic. Moreover, treatment with $125-500 \mathrm{pg} / \mathrm{ml}$ PYP1 increased cell viability from $114.2 \pm 12.5$ to $145.0 \pm 28.2 \%(\mathrm{P}<0.05)$ compared with the control. To determine whether PYP1 protects the Chang liver cells against APAP-induced death, cell viability was examined following treatment with APAP. The cells treated with $15 \mathrm{mM}$ APAP demonstrated a viability of $76.6 \pm 13.3 \%$. By contrast, when the cells were treated with $125-500 \mathrm{pg} / \mathrm{ml}$ PYP1, cell viability increased significantly to $127.0 \pm 7.8,110.2 \pm 15.6$ and $91.0 \pm 4.6 \%$ that of the control, respectively (Fig. 4B, P<0.05). The same result was noted in the cells stained with DAPI, according to fluorescence microscopy (Fig. 4C). Similar results were obtained for both PYP1-AC and PYP1-B (Figs. 5 and 6). No significant differences were observed with regard to cytotoxicity by PYP1-AC $(\mathrm{P}<0.05$, Fig. $5 \mathrm{~A})$; however, cell viability increased significantly following treatment with $125-500 \mathrm{pg} / \mathrm{ml}$ PYP1-AC to $102.7 \pm 14.5,103.5 \pm 7.6$ and $111.7 \pm 9.3 \%$ compared to that of the control, respectively $(\mathrm{P}<0.05$, Fig. $5 \mathrm{~B})$. These results are presented as photomicrographic images in Fig. 5C. Cell viability increased significantly to $151.4 \pm 13.6 \%$ following treatment with $125-1000 \mathrm{pg} / \mathrm{ml} \mathrm{PYP} 1-\mathrm{B}$ ( $\mathrm{P}<0.05$, Fig. 6A). In addition, treatment with PYP1-B resulted in the highest value $(151.4 \pm 12.6 \%)$ of all the APAP treatment groups $(\mathrm{P}<0.05$,

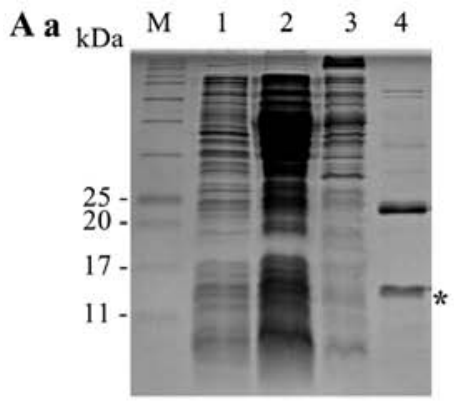

b

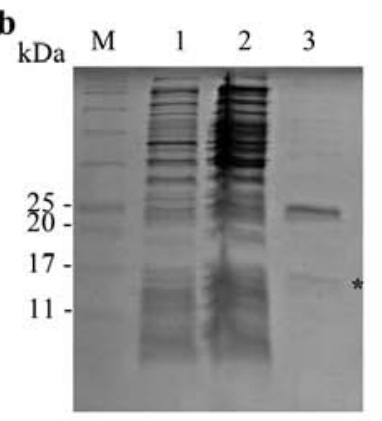

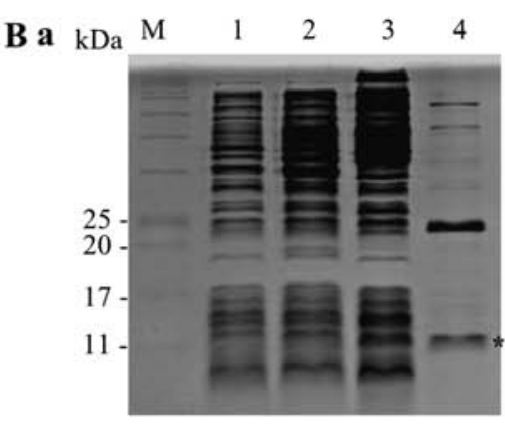

b

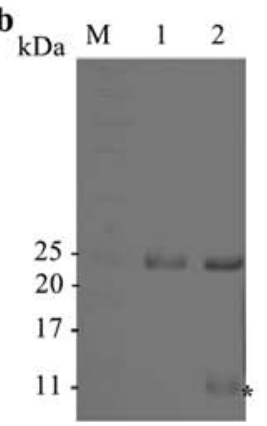



b

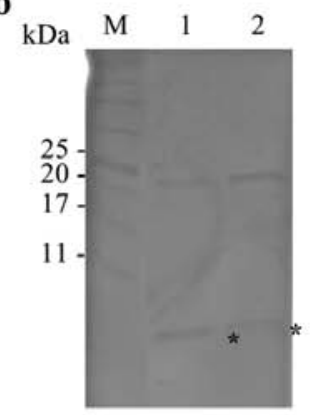

Figure 3. SDS-PAGE analysis of recombinant PYP1s expressed in the E. coli strain BL21. Samples were resolved by $17 \%$ SDS-PAGE. Recombinant proteins are indicated by an asterisk. (A) Recombinant PYP1. (a) M, protein marker; lane 1, before IPTG induction; lane 2, after IPTG induction; lane 3, insoluble protein; lane 4, first purified, soluble protein; (b) M, protein marker; lane 1, before IPTG induction; lane 2, after IPTG induction; lane 3, second purified, soluble protein. (B) Recombinant PYP1-AC. (a) M, protein marker; lane 1, before IPTG induction; lane 2, after IPTG induction; lane 3; soluble protein; lane 4, first purified, soluble protein; (b) M, protein marker; lane 1, control vector; lane 2, second purified, soluble protein. (C) Recombinant PYP1-B. (a) M, protein marker; lane 1, before IPTG induction; lane 2, after IPTG induction; lane 3, soluble protein; lane 4, insoluble protein; lane 5, first purified, soluble protein fraction 1; lane 6, first purified, soluble protein fraction 2; (b) M, protein marker; lanes 1 and 2, second purified, soluble protein. PYP1, Pyropia yezoensis protein 1. 


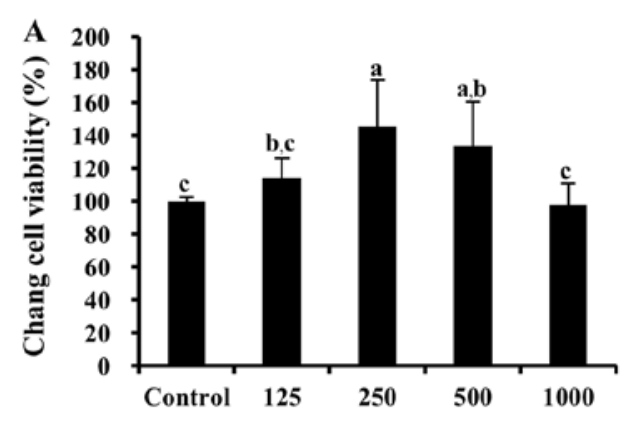

Concentration of PYP1 (pg/ml)
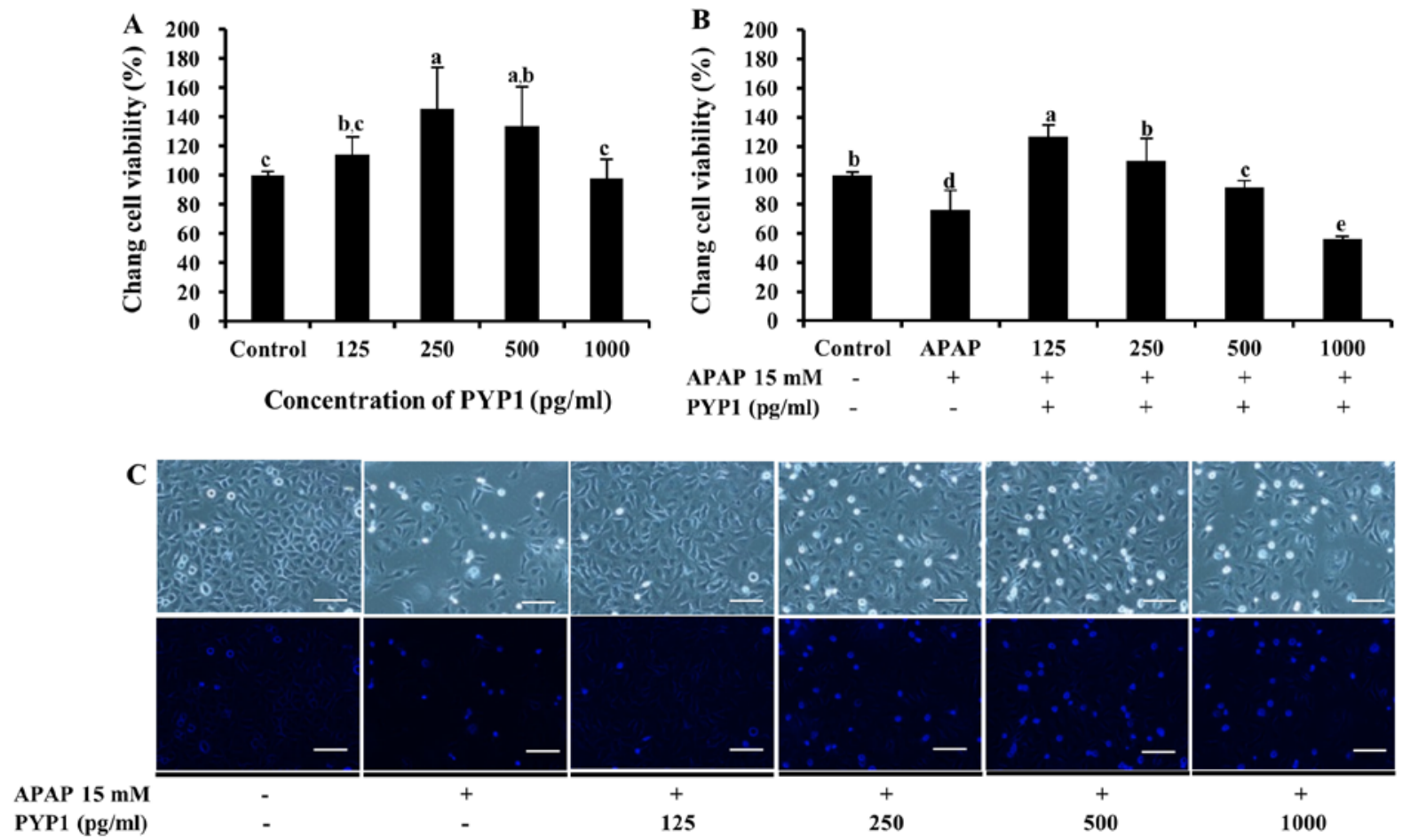

Figure 4. (A) Recombinant Pyropia yezoensis protein 1 (PYP1) toxicity in Chang liver cells. (B) Protective effects of PYP1 on acetaminophen (APAP)-induced liver damage. Cell viability was measured by MTS assays. Values represent the means \pm standard deviation (SD) (P<0.05). Bars labeled with different letters indicate significant differences among groups, as determined by Duncan's multiple-range test. (C) Morphological changes in cells following treatment with APAP alone or APAP + PYP1. Upper and lower panels show bright field and fluorescence images. Scale bar, $100 \mu \mathrm{m}$.

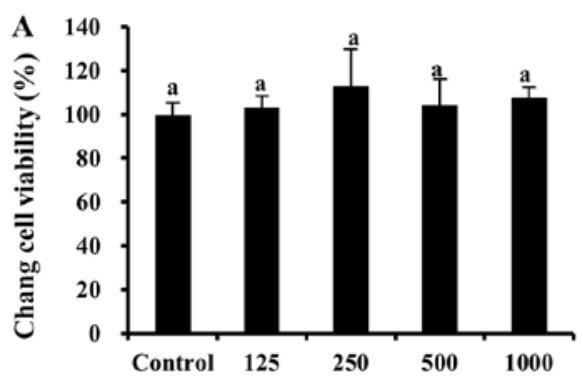

Concentration of PYP1-AC (pg/ml)

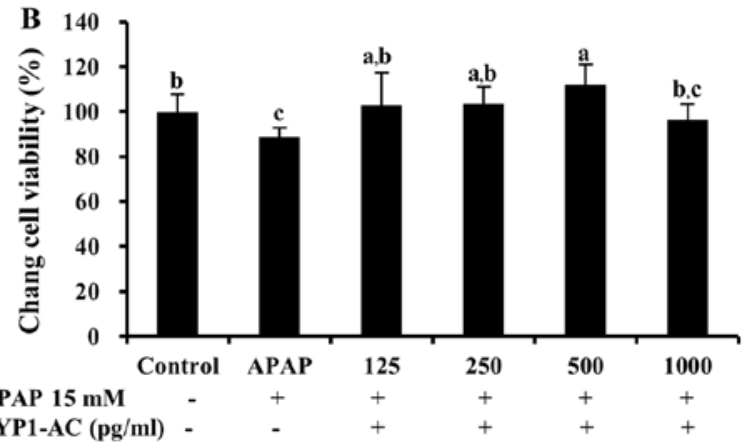

C
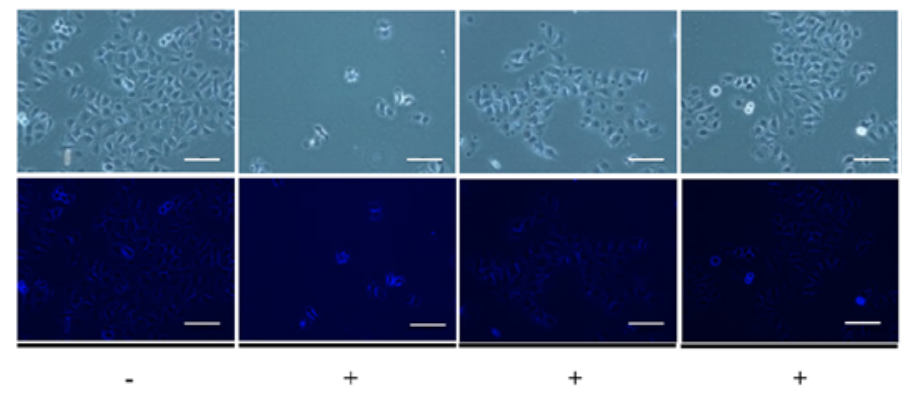

250

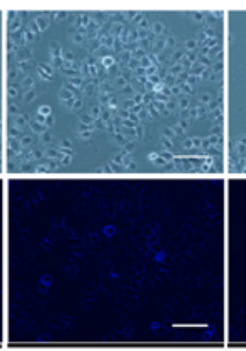

$+$

500

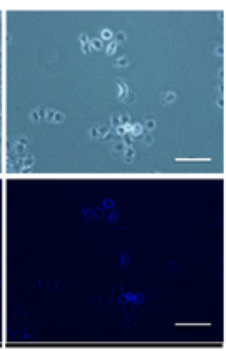

$+$

1000

Figure 5. (A) Recombinant Pyropia yezoensis protein 1 (PYP1)-AC toxicity in Chang liver cells. (B) Protective effects of PYP1-AC on acetaminophen (APAP)-induced liver damage. Cell viability was measured by MTS assays. Values represent the means $\pm \mathrm{SD}(\mathrm{P}<0.05)$. Bars labeled with different letters indicate significant differences among groups, as determined by Duncan's multiple-range test. (C) Morphological changes in cells following treatment with APAP alone or APAP + PYP1-AC. Upper and lower panels show bright field and fluorescence images. Scale bar, $100 \mu \mathrm{m}$.

Fig. 6B). The effect of PYP1-B was also demonstrated by examining cellular morphology (Fig. 6C). These results suggest that recombinant PYP1s may be used to protect liver cells against APAP-induced cytotoxicity. 


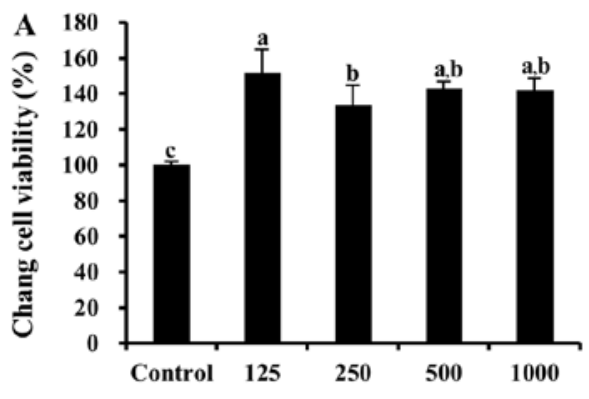

Concentration of PYP1-B (pg/ml)

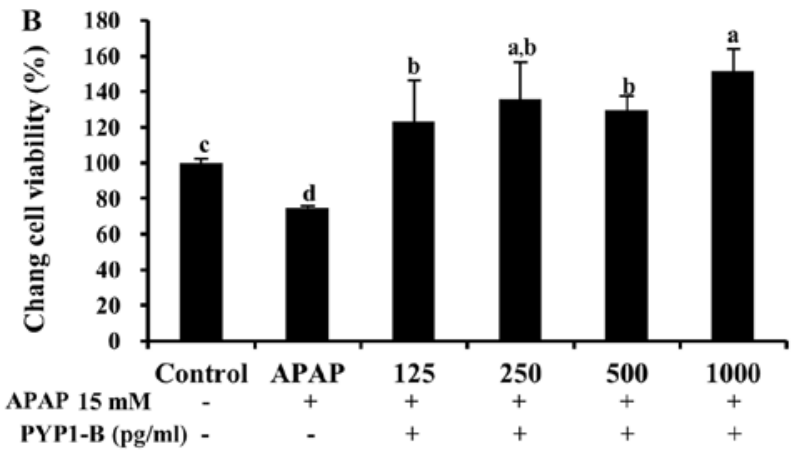

PYP1-B (pg/ml) -

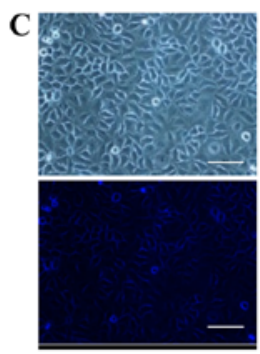

APAP $15 \mathrm{mM}$

PYP1-B (pg/ml)

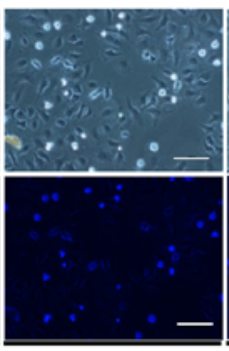

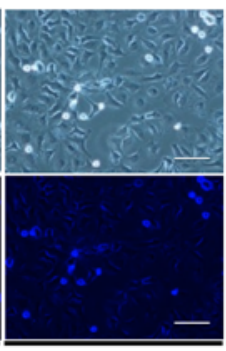

125
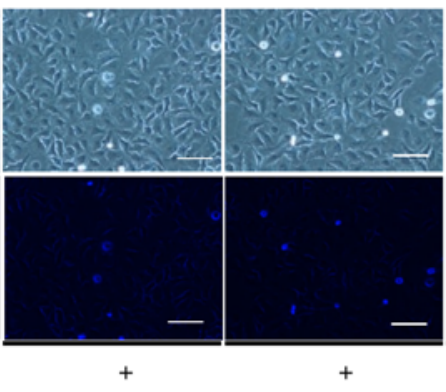

250

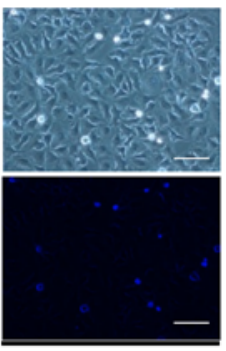

500
1000

Figure 6. (A) Recombinant Pyropia yezoensis protein 1 (PYP1)-B toxicity in Chang liver cells. (B) Protective effects of PYP1-B on acetaminophen (APAP)-induced liver damage. Cell viability was measured by MTS assays. Values represent the means $\pm \mathrm{SD}(\mathrm{P}<0.05)$. Bars labeled with different letters indicate significant differences among groups,as determined by Duncan's multiple-range test. (C) Morphological changes in cells following treatment with APAP alone or APAP + PYP1-B. Upper and lower panels show bright field and fluorescence images. Scale bar, $100 \mu \mathrm{m}$.


Figure 7. (A) Synthetic peptide (SP) toxicity in Chang liver cells. (B) Protective effects of SP on acetaminophen (APAP)-induced liver damage. Cell viability was measured by MTS assays. Values represent the means $\pm \mathrm{SD}(\mathrm{P}<0.05)$. Bars labeled with different letters indicate significant differences among groups, as determined by Duncan's multiple-range test. (C) Morphological changes in cells following treatment with APAP alone or APAP + SP. Upper and lower panels show bright field and fluorescence images. Scale bar, $100 \mu \mathrm{m}$.

Physiological activity of SP. To determine whether SP, which contains the $\mathrm{N}$-terminal 11-residue sequence, protects
Chang liver cells against APAP-induced death, cell viability was examined following treatment with APAP. As shown in 
Fig. 7A, SP was not toxic to the cells and resulted in cellular proliferation following treatment with $125-500 \mathrm{pg} / \mathrm{ml} \mathrm{SP}$. Notably, following treatment with both APAP and SP, cell viability decreased significantly compared with that of the control $(\mathrm{P}<0.05$, Fig. 7B). Lower values upon SP treatment (64.9 \pm 7.5 to $75.9 \pm 7.5 \%, 125-1,000 \mathrm{pg} / \mathrm{ml} \mathrm{SP})$ compared to those observed with PYP1, PYP1-AC and PYP1 were observed in the APAP-treated cells. However, treatment with SP led to a significant increase in cell viability compared to treatment with APAP alone $(61.8 \pm 8.6 \%$; $\mathrm{P}<0.05$, Fig. $7 \mathrm{~B})$. The cells treated with SP appeared to increase in number compared to the cells treated with APAP only (Fig. 7C).

\section{Discussion}

In a previous study of ours (18), we suggested that PYP1, a novel protein from red alga $P$. yezoensis, had a sequence homology with that of hypothetical, unknown proteins from Chondrus crispus (Rhodophyta) and Emiliania huxleyi (Haptophyceae). The physicochemical characteristics of PYP1 are similar to those of late embryogenesis abundant (LEA) proteins. LEA proteins protect against protein denaturation caused by various environmental factors, such as desiccation, freezing, heat, salt and osmotic stress (20). Since P. yezoensis is one of the marine red algae that regulate environmental stress responses, it is frequently used to study molecular mechanisms (21).

Although APAP is an over-the-counter drug that is widely used for analgesic and antipyretic purposes, an overdose can cause liver injury, liver failure and even death (22). For these reasons, a variety of studies have investigated APAP metabolism in the liver, and various protective factors against APAP-induced liver toxicity, such as genistein (23) and manganese superoxide dismutase (SOD) (24), have been noted. Few researchers have studied the mechanisms of action of PYP since Hwang et al (8) reported the effects of PYP against APAP-induced liver cell injury. Currently, only one study using a particular PYP peptide has reported its regulation of multiple cell growth-related signals in MCF-7 cells (25). PYP has also been shown to stimulate the proliferation of IEC-6 normal intestinal epithelial cells and is associated with the insulinlike growth factor I (IGF-IR) and epidermal growth factor receptor (EGFR) signaling pathways $(26,27)$.

In the present study, three PYP1 proteins and one SP containing the N-terminal 11-residue of the PYP1s were used to investigate the chemoprotective activities of recombinant PYP1s against APAP-induced cytotoxicity. We noted that SP, as well as PYP1 proteins, exhibited highly positive and supportive chemoprotective activities against APAP-induced toxicity. In addition, SP had a similar effect against methotrexate-induced cell death in Chang liver cells (data not shown). Therefore, these results suggest that the PYP peptide exerts protective effects against liver cell injury.

In general, macroalgae represent ideal starting materials for the generation of marine protein-derived bioactive peptides due to their high protein content (28). As these bioactive peptides act on numerous physiological functions (29), we should focus on macroalgae from natural materials. These peptides are involved in the modulation of cell proliferation-associated molecules (30). In addition, peptides from macroalgae have been reported to possess several biological traits, including ACE inhibitory and anti-hypertensive effects $(31,32)$. Of note, Suetsuna and Saito (33) reported that PYP inhibits antimutagenesis and $\mathrm{Ca}^{2+}$ precipitation, lowers plasma and hepatic cholesterol, improves hepatic function, reduces blood sugar and exhibits antioxidant and SOD-like qualities; PYP also has angiotensin-convertingenzyme (ACE) inhibitory and anti-hypertensive effects. Our findings demonstrated that PYP1s, as bioactive peptides, have a positive effect against hepatic toxicity. However, the potential of this bioactive peptide in vivo remains to be elucidated; a specific biological activity observed in vitro is only an indicator of the potential active component in vivo (28). Therefore, further studies are required to address the biological activity of PYP1s in vivo and in signaling pathways.

\section{Acknowledgements}

This study was supported by the Basic Science Research Program through the National Research Foundation of Korea (NRF), funded by the Ministry of Education (2012R1A6A1028677). We are grateful to Ms. Megumi Akita for her technical assistance.

\section{References}

1. Mohamed S, Hashim SN and Rahman HA: Seaweeds: A sustainable functional food for complementary and alternative therapy. Trends Food Sci Technol 23: 83-96, 2012.

2. Maeda H, Hosokawa M, Sashima T, Funayama K and Miyashita K: Fucoxanthin from edible seaweed, Undaria pinnatifida, shows antiobesity effect through UCP1 expression in white adipose tissues. Biochem Biophys Res Commun 332: 392-397, 2005.

3. Colliec S, Fischer AM, Tapon-Bretaudiere J, Boisson C, Durand P and Jozefonvicz J: Anticoagulant properties of a fucoidan fraction. Thromb Res 64: 143-154, 1991.

4. Funahashi H, Imai T, Mase T, Sekiya M, Yokoi K, Hayashi H, Shibata A, Hayashi T, Nishikawa M, Suda N, et al: Seaweed prevents breast cancer? Jpn J Cancer Res 92: 483-487, 2001.

5. Hwang HJ, Kim IH and Nam TJ: Effect of a glycoprotein from Hizikia fusiformis on acetaminophen-induced liver injury. Food Chem Toxicol 46: 3475-3481, 2008a.

6. Choi EY, Hwang HJ, Kim IH and Nam TJ: Protective effects of a polysaccharide from Hizikia fusiformis against ethanol toxicity in rats. Food Chem Toxicol 47: 134-139, 2009.

7. Shin ES, Hwang HJ, Kim IH and Nam TJ: A glycoprotein from Porphyra yezoensis produces anti-inflammatory effects in liposaccharide-stimulated macrophages via the TLR4 signaling pathway. Int J Mol Med 28: 809-815, 2011.

8. Hwang HJ, Kwon MJ, Kim IH and Nam TJ: Chemoprotective effects of a protein from the red algae Porphyra yezoensis on acetaminophen-induced liver injury in rats. Phytother Res 22: 1149-1153, 2008b.

9. Lee WM: Acetaminophen and the U.S. Acute Liver Failure Study Group: Lowering the risks of hepatic failure. Hepatology 40: 6-9, 2004.

10. Shon YH and Nam KS: Protective effect of moutan cortex extract on acetaminophen-induced cytotoxicity in human Chang liver cells. Biol Pharm Bull 25: 1427-1431, 2002.

11. Rajaraman G, Chen J and Chang TKH: Ginkgolide A contributes to the potentiation of acetaminophen toxicity by Ginkgo biloba extract in primary cultures of rat hepatocytes. Toxicol Appl Pharmacol 217: 225-233, 2006.

12. Hwang HJ, Kwon MJ and Nam TJ: Chemoprotective effect of insulin-like growth factor I against acetaminophen-induced cell death in Chang liver cells via ERK1/2 activation. Toxicology 230: 76-82, 2007.

13. Gong Y, Wang G, Gong Y, Yan J, Chen Y and Burczynski FJ: Hepatoprotective role of liver fatty acid binding protein in acetaminophen induced toxicity. BMC Gastroenterol 14: 44, 2014.

14. Ray SD, Kumar MA and Bagchi D: A novel proanthocyanidin IH636 grape seed extract increases in vivo Bcl-XL expression and prevents acetaminophen-induced programmed and unprogrammed cell death in mouse liver. Arch Biochem Biophys 369: 42-58, 1999. 
15. Cover C, Fickert P, Knight TR, Fuchsbichler A, Farhood A, Trauner $M$ and Jaeschke $H$ : Pathophysiological role of poly(ADP-ribose) polymerase (PARP) activation during acetaminophen-induced liver cell necrosis in mice. Toxicol Sci 84 : 201-208, 2005.

16. Oliveira FA, Chaves MH, Almeida FRC, Lima RCP Jr, Silva RM, Maia JL, Brito GAAC, Santos FA and Rao VS: Protective effect of $\alpha$ - and $\beta$-amyrin, a triterpene mixture from Protium heptaphyllum (Aubl.) March. trunk wood resin, against acetaminophen-induced liver injury in mice. J Ethnopharmacol 98 103-108, 2005

17. Raghavendran HRB, Sathivel A and Devaki T: Protective effect of Sargassum polycystum (brown alga) against acetaminopheninduced lipid peroxidation in rats. Phytother Res 19: 113-115, 2005.

18. Choi YH, Yamaguchi K, Oda T and Nam TJ: Chemical and mass spectrometry characterization of the red alga Pyropia yezoensis chemoprotective protein (PYP): Protective activity of the $\mathrm{N}$-terminal fragment of PYP1 against acetaminophen-induced cell death in Chang liver cells. Int J Mol Med 35: 271-276, 2015.

19. Uji T, Hirata R, Mikami K, Mizuta $\mathrm{H}$ and Saga N: Molecular characterization and expression analysis of sodium pump genes in the marine red alga Porphyra yezoensis. Mol Biol Rep 39: 7973-7980, 2012.

20. Battaglia M, Olvera-Carrillo Y, Garciarrubio A, Campos F and Covarrubias AA: The enigmatic LEA proteins and other hydrophilins. Plant Physiol 148: 6-24, 2008.

21. Blouin NA, Brodie JA, Grossman AC, Xu P and Brawley SH: Porphyra: a marine crop shaped by stress. Trends Plant Sci 16: 29-37, 2011.

22. Xie Y, McGill MR, Dorko K, Kumer SC, Schmitt TM, Forster J and Jaeschke H: Mechanisms of acetaminophen-induced cell death in primary human hepatocytes. Toxicol Appl Pharmacol 279: 266-274, 2014.

23. Fan YJ, Rong Y, Li PF, Dong WL, Zhang DY, Zhang L and Cui MJ: Genistein protection against acetaminophen-induced liver injury via its potential impact on the activation of UDP-glucuronosyltransferase and antioxidant enzymes. Food Chem Toxicol 55: 172-181, 2013.
24. Ramachandran A, Lebofsky M, Weinman SA and Jaeschke H: The impact of partial manganese superoxide dismutase (SOD2)deficiency on mitochondrial oxidant stress, DNA fragmentation and liver injury during acetaminophen hepatotoxicity. Toxicol Appl Pharmacol 251: 226-233, 2011.

25. Park SJ, Ryu J, Kim IH, Choi YH and Nam TJ: Induction of apoptosis by a peptide from Poryphyra yezoensis: Regulation of the insulin-like growth factor I receptor signaling pathway in MCF-7 cells. Int J Oncol 45: 1011-1016, 2014

26. Lee MK, Kim IH, Choi YH and Nam TJ: A peptide from Porphyra yezoensis stimulates the proliferation of IEC-6 cells by activating the insulin-like growth factor I receptor signaling pathway. Int J Mol Med 35: 533-538, 2015.

27. Lee MK, Kim IH, Choi YH, Choi JW, Kim YM and Nam TJ: The proliferative effects of Pyropia yezoensis peptide on IEC-6 cells are mediated through the epidermal growth factor receptor signaling pathway. Int J Mol Med 35: 909-914, 2015.

28. Harnedy PA and FitzGerald RJ: Bioactive proteins, peptides, and amino acids from macroalgae. J Phycol 47: 218-232, 2011.

29. Murray BA and FitzGerald RJ: Angiotensin converting enzyme inhibitory peptides derived from food proteins: Biochemistry, bioactivity and production. Curr Pharm Des 13: 773-791, 2007.

30. Morse ANC: GABA-mimetic peptides form marine algae and cyanobacteria as potential diagnostic and therapeutic agents. In: Bioactive Compounds From Marine Organisms - With emphasis on the Indian Ocean. Thompson MF, Sarojini R and Nagabhushanam R (eds). Oxford \& IBH Publishing Co., New Delhi, India, pp167-172, 1991.

31. Suetsuna K: Purification and identification of angiotensin I-converting enzyme inhibitors from the red alga Porphyra yezoensis. J Mar Biotechnol 6: 163-167, 1998.

32. Saito M and Hagino H: Antihypertensive effect of oligopeptides derived from nori (Porphyra yezoensis) and Ala-Lys-Tyr-Ser-Tyr in rats. J Jpn Soc Nutr Food Sci 58: 177-184, 2005.

33. Suetsuna K and Saito M: Enzyme-decomposed materials of laver and uses thereof. US Patent 6217879B1, April 17, 2001. 